\title{
Micorrizas arbusculares y hongos septados oscuros nativos en topinambur (Helianthus tuberosus L.) en Catamarca, Argentina
}

\author{
Arbuscular mycorrhizal and native dark septate fungus in jerusalem artichoke (Helianthus \\ tuberosus L.) in Catamarca, Argentina
}

\section{Gabriela Di Barbaro1; Horacio Andrada²; Valeria González Basso33; Ana Lilia Alurralde4; Eleodoro Del Valle ${ }^{5}$; Celia Brandán de Weht ${ }^{6}$}

1. Especialista. Universidad Nacional de Catamarca. Argentina, gabydibarbaro@yahoo.com.ar.

2. Ingeniero Agrónomo. Universidad Nacional de Catamarca. Argentina, horacio_andrada@yahoo.com.ar.

3. Ingeniero Agrónomo. Universidad Nacional de Catamarca. Argentina, valegb82@hotmail.com.

4. Ingeniero Agrónomo. Universidad Nacional de Catamarca. Argentina, ani_animal@hotmail.com.

5. DSc. Universidad Nacional del Litoral. Santa Fe. Argentina, eleodoro77@gmail.com.

6. DSc. Universidad Nacional de Tucumán. Argentina, celiabrandan@gmail.com.

Citar: Di Barbaro, G.; Andrada, H.; Gonzalez, V.; Alurralde, A.; Del Valle, E.; Brandran, C. 2017. Micorrizas arbusculares y hongos septados oscuros nativos en topinambur (Helianthus tuberosus L.) en Catamarca, Argentina. Rev. Cienc. Agri. 34(2): 98 - 106. doi: http://dx.doi.org/10.22267/rcia.173402.75.

Recibido: Julio 18 de 2016. Aceptado: Octubre 10 de 2017.

\section{RESUMEN}

Para determinar y valorar la infección fúngica radicular, se estudió la colonización por simbiontes fúngicos nativos en raíces de Topinambur (Helianthus tuberosus L.), cultivado en el Valle Central de la provincia de Catamarca, región árida del Noroeste Argentino. Los estudios se efectuaron mediante observación microscópica de estructuras fúngicas teñidas dentro de la raíz. La cuantificación de la infección se efectuó mediante metodologías de clarificación y conteo sobre cuadricula bajo lupa. Se observaron estructuras endomicorrícicas como hifas, arbúsculos y vesículas del tipo vesículo arbuscular (MVA) y de hongos endofíticos septados oscuros (ESO) con hifas tabicadas, melanizadas y con numerosos microesclerocios. Se determinó un alto nivel de colonización fúngica con ocurrencia simultánea de MVA y hongos ESO. Este estudio es el primer informe sobre colonización por MVA y ESO en raíces de topinambur.

Palabras claves: hongos de raíz, endomicorrizas, alcachofa de Jerusalén, ESO. 


\section{ABSTRACT}

In order to determine and evaluate the fungal root infection it was studied the colonization by native fungal symbionts in roots of jerusalem artichoke (Helianthus tuberosus L.) cultivated in the Central Valley of the province of Catamarca, an arid region at the Northwest of Argentina. The studies were made by: microscope observation of the stained root fungal structures. The infection quantification was made by clarification methodologies and count under magnifying glass. Endomycorrhizal structures of the vesicle arbuscular type were observed (MVA; hyphae, arbuscules and vesicles) and of septate dark endophytic fungi (DES; melanized septate hyphae and with numerous microesclerotia). A high level of fungi colonization was determined with simultaneous occurrence of vesicle arbuscular mycorrhizae (VAM) and fungi (DES). This study is the first report of the colonization by VAM and DES in roots of jerusalem artichoke.

Key words: root fungi, endomycorrhizas, jerusalem artichoke, DES.

\section{INTRODUCCIÓN}

La simbiosis micorrícico-arbuscular (MA) se establece entre las raíces de la mayoría de las plantas terrestres y hongos pertenecientes al Phylum Glomeromycota (Schübler et al., 2001). Estos hongos reciben compuestos carbonados de la planta, como contraparte estos promueven el crecimiento de las plantas al suministrar nutrientes del suelo especialmente los pocos móviles como fósforo y agua (Kirk et al., 2001; Harrison, 2005; Selosse et al., 2006; Wang y Qiu, 2006; Parodi y Pezzani, 2011). Además, confieren una mayor tolerancia al ataque de patógenos y a la sequía (Newsham et al., 1995; Pérez y Vertel, 2010). Las micorrizas constituyen un recurso biológico cuyo manejo y conservación, genera beneficios ambientales al mejorar las condiciones físico-químicas y biológicas del suelo (Pérez y Vertel, 2010; Martínez, 2011).

Es frecuente encontrar un grupo de Ascomycetes anamórficos dematiáceos que colonizan intra e intercelularmente los tejidos de las raíces formando asociaciones que van desde el mutualismo al parasitismo (Jumpponen, 2001). Estos hongos, denominados Endófitos Septados Oscuros (ESO), se asocian con angiospermas dicotiledóneas y monocotiledóneas, y se encuentran a menudo en las plantas micorrizadas. Los ESO son en su mayoría pigmentados y crecen en el parénquima cortical y en elementos de vaso del cilindro vascular central. Forman microesclerocios pigmentados, de estructura hifal apretada y sinuosa (Jumpponen y Trappe, 1998). Además, presentan hifas hialinas y delgadas con cuerpos lipídicos en su interior, denominadas SEF-(systemic endophytic fungi), (Barrow, 2003), que colonizan en forma sistémica al hospedante. Estas hifas incoloras constituyen zonas potenciales de transferencia de carbono (Barrow y Aaltonen, 2001).

El topinambur o alcachofa de Jerusalén (Helianthus tuberosus L.) es una Asterácea originaria de América Central (Cosgrove et al., 1991). En Argentina fue introducido a principios del siglo XX para los programas de mejoramiento de la especie y también de girasol (Helianthus annuus L.), donde el topinambur constituye una fuente de genes de interés agronómico (Rebora, 2008). Se cultiva como hortaliza, forrajera, industrial y medicinal, sin embargo en Catamarca sólo se cultiva para la alimentación animal, fundamentalmente para producción porcina. Uno de los primeros usos fue en investigaciones histológicas, debido a las características de sus tallos, que han sido utilizados para el 
acondicionamiento de material fitopatológico con la finalidad de realizar cortes finos y precisos de material fresco para que faciliten su observación (Sarasola y Rocca, 1975).

Los antecedentes sobre micorrizas en plantas del género Helianthus se refieren fundamentalmente a la inoculación para mejorar el rendimiento del cultivo de Girasol (Helianthus annus L.) (Álvarez et al., 2008; Perez, 2012). Debido a que no se registran antecedentes que estudien el mutualismo de hongos benéficos asociados con plantas de topinambur en la provincia de Catamarca ni en el país, los objetivos de esta investigación fueron estudiar los hongos formadores de micorrizas arbusculares u otros tipos de hongos y la incidencia de los mismos en Helianthus tuberosus en el Valle Central de la provincia de Catamarca, República Argentina.

\section{MATERIALES Y MÉTODOS}

Material vegetal. Muestras de plantas de topinambur (Helianthus tuberosus L. variedad Topianka) de 90 días en floración.

Localización. Procedencia de plantas de topinambur de un cultivo a campo, ubicado en el Valle Central de la provincia de Catamarca a (28 $\left.28^{\prime} 07^{\prime \prime} \mathrm{LS} 65^{\circ} 46^{\prime} 60^{\prime \prime} \mathrm{LO}\right)$, que está inmerso en la región semiárida del noroeste de Argentina.

Tratamiento de raíces. Las muestras de raíces se recolectaron al azar de cinco plantas en el período de plena floración. En laboratorio, las raíces de cada unas de las plantas recolectadas se extrajeron y lavaron con agua corriente, luego se seleccionaron las más delgadas, las que se clarificaron y tiñeron siguiendo la metodología de Phillips y Hayman (1970) para la detección de estructuras de micorrizas (Figura 1). La tinción se realizó con solución de triple colorante de Gueguén (Sarasola y Rocca, 1975), porque permite diferenciar algunos elementos del hongo las grasas aparecen en color rosa o en rojo, el glicógeno en caoba, el almidón en azul (Verna y Herrero, 1952).

Examen microscópico. Las raíces se montaron entre porta y cubreobjetos, por cada ejemplar se realizaron preparados microscópicos. Las raíces fueron observadas utilizando microscopio óptico con objetivos estándar de 10,40 y $100 x$.

Cuantificación de infección. Para cada individuo se cuantificó el porcentaje de colonización por MVA (hifas, arbúsculos y vesículas) y ESO. Se realizó la cuantificación microscópica del porcentaje de colonización micorrícica arbuscular (MA), el contenido de arbúsculos (A) y de vesículas (V), por el método de intersección de línea de Giovannetti y Mosse (1980). Para ello, de cada ejemplar se tomaron 15 segmentos de las raíces tratadas y coloreadas. Se distribuyeron al azar sobre un portaobjeto cuadriculado y mediante observación microscópica (10x y 40x) se registró la presencia-ausencia de estructuras micorrícicas (A y V) en las intersecciones horizontales y verticales entre raíces y líneas de la cuadrícula (Figura 2). De cada planta se realizaron tres repeticiones, cuantificándose al menos 100 intersecciones por preparado y se calculó la frecuencia de infección micorrícica, $\mathrm{M}_{\text {Giovannetti }}(\%)=(\mathrm{SI} \times 100) / \mathrm{SO}$, donde SI corresponde al número de segmentos infectados (hifas + arbúsculos + vesículas) y SO al número de segmentos observados totales (hifas + arbúsculos + vesículas + sin infección). También se calculó la frecuencia de aparición de arbúsculos y de vesículas (Covacevich et al., 2001), cuantificándose arbúsculos, vesículas e hifas de micorrizas VA, además se registró el porcentaje de hifas de ESO y porcentaje de microesclerocios en raicillas de Topinambur. 


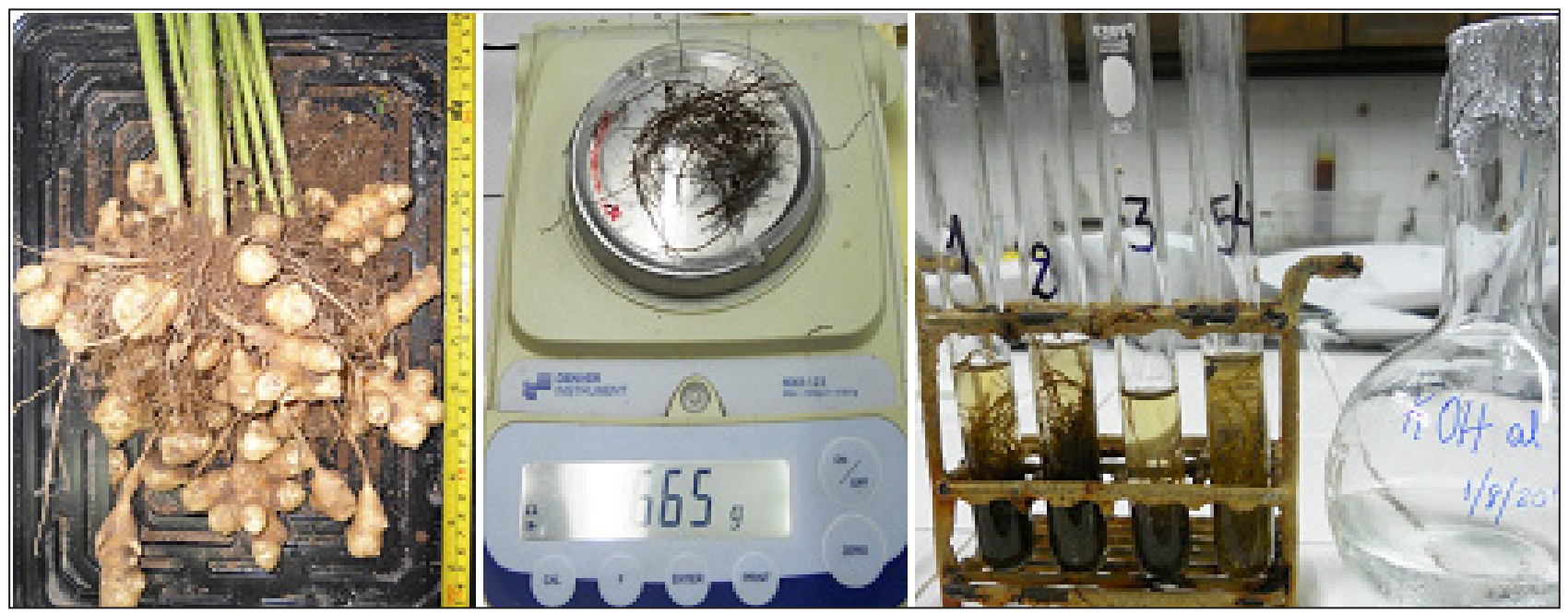

Figura 1. Técnica de clareo para la observación de estructuras de micorrizas en raíces de Topinambur (Helianthus tuberosus L.).

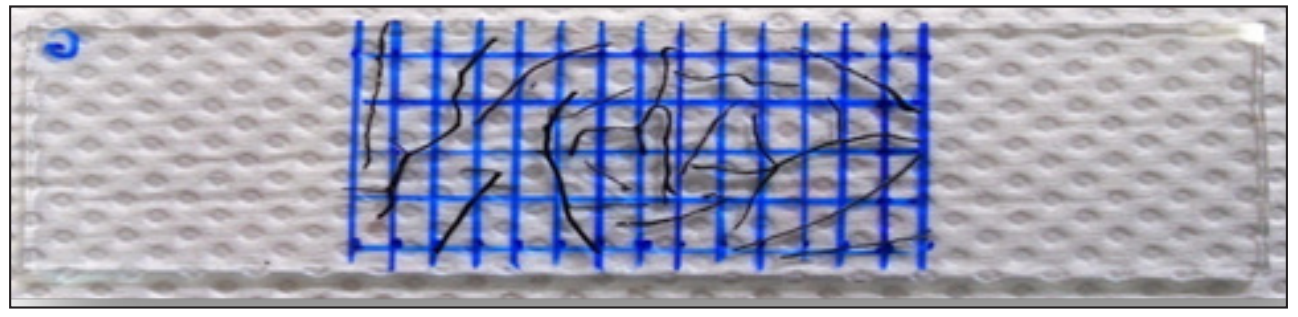

Figura 2. Raicillas de Topinambur (Helianthus tuberosus L.) sobre cuadrícula de portaobjeto para determinar el grado de colonización micorrícica por el método de intersección de línea de Giovannetti y Mosse (1980).

\section{RESULTADOS Y DISCUSIÓN}

Tres estructuras típicas de las micorrizas vesículo-arbusculares (VA) fueron observadas: hifas, arbúsculos y vesículas, en raíces de todas las plantas de Topinambur recolectadas. (Figura 3). Las hifas son continuas finas y gruesas, algunas de ellas con lípidos en rosario en su interior con creci- miento intracelular e intercelular. Se destaca la presencia de una raíz con arbúsculos bien desarrollados. También se observaron numerosas vesículas de morfología diversa (esféricas, ovaladas, ahusadas) (Figura 3 y 4), por lo que se infiere que las raicillas de este vegetal están colonizadas por varias especies o géneros de hongos micorrícicos VA nativos. 


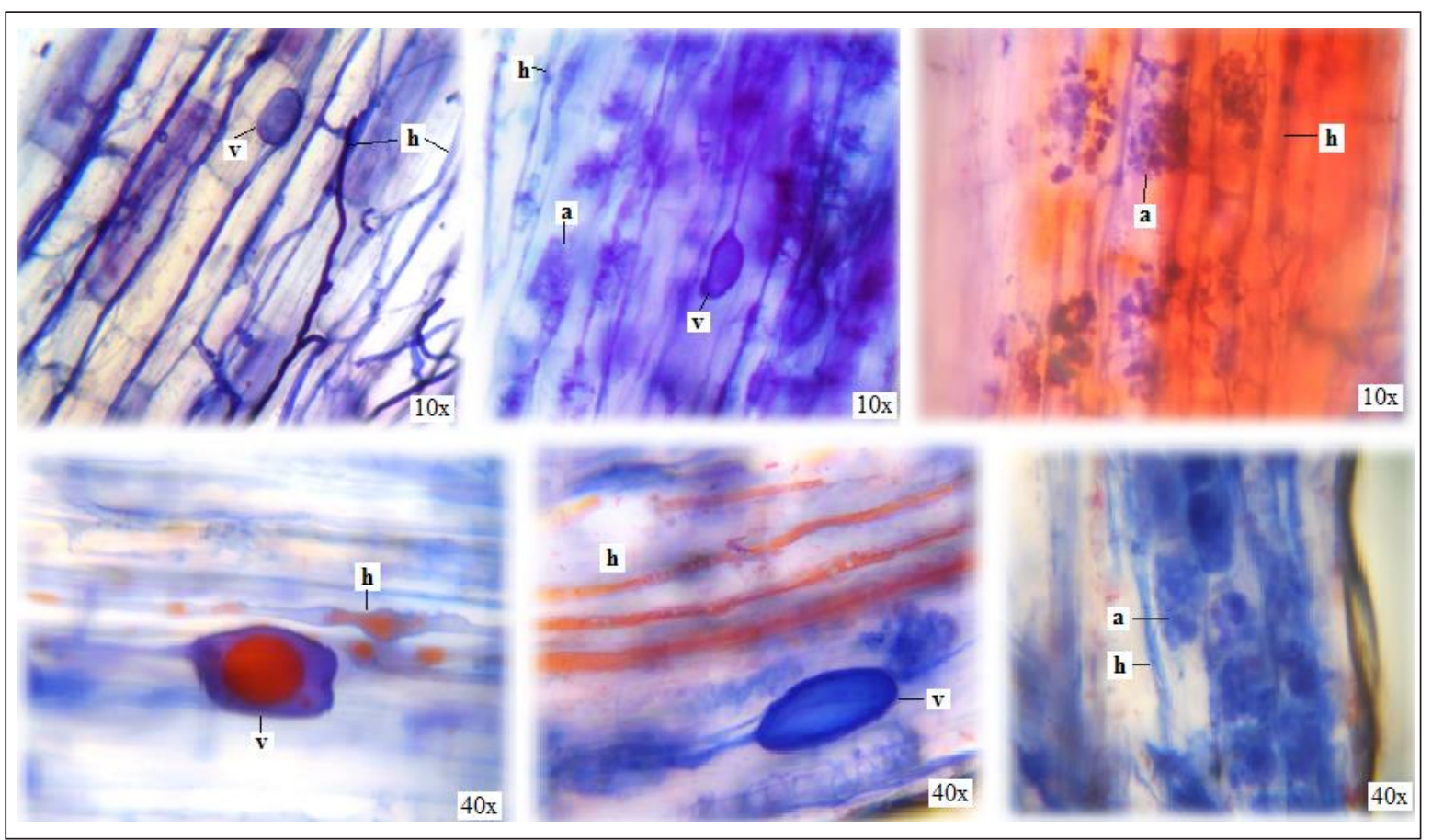

Figura 3. Micrografías de estructuras de micorrizas en raíces de topinambur (Helianthus tuberosus L.) a: arbúsculos; h: hifas; v: vesículas.

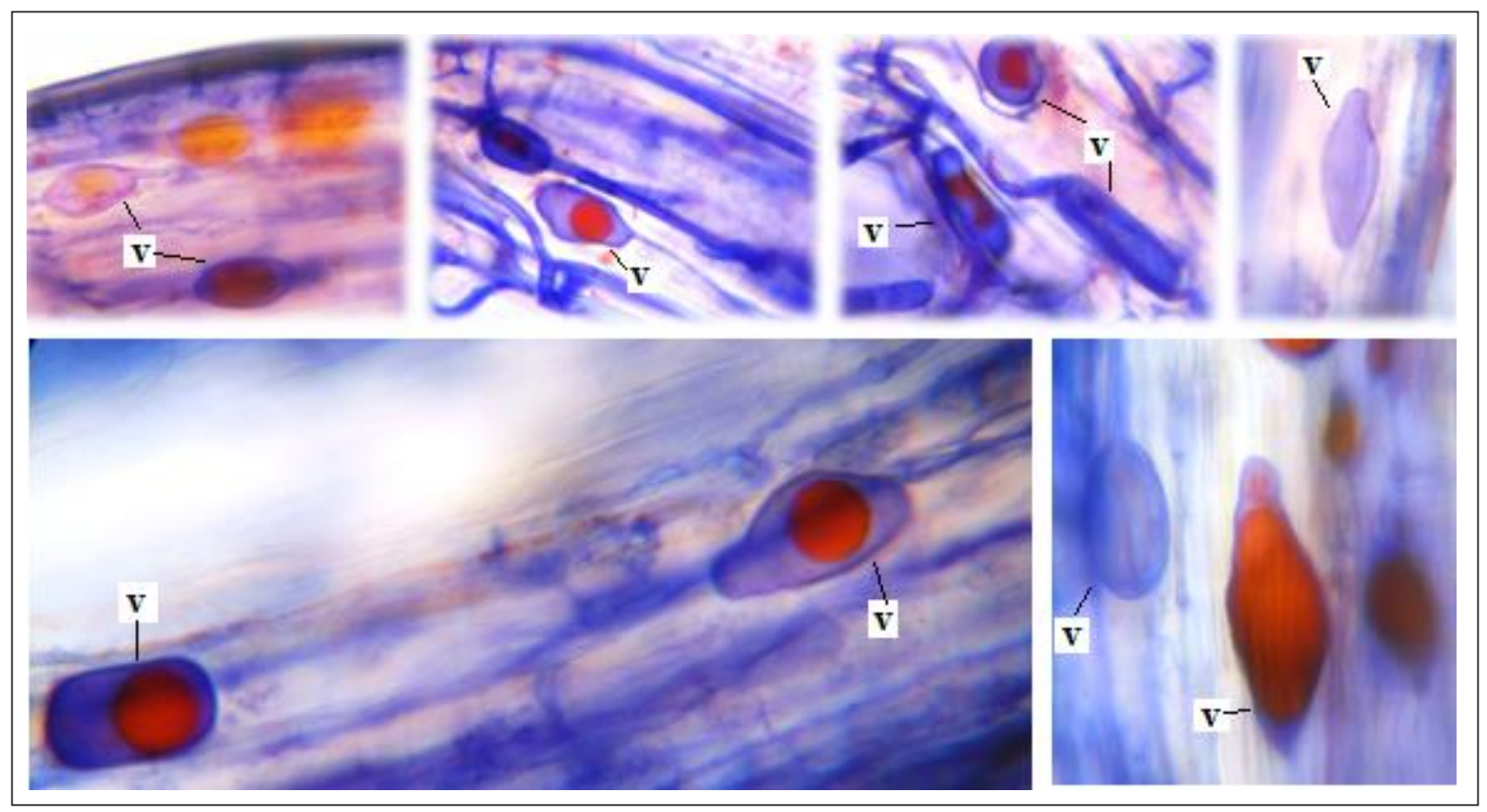

Figura 4. Micrografías (40x) de vesículas de diversas formas en la misma raicilla de Topinambur (Helianthus tuberosus L.). 
Las vesículas se mostraron con coloraciones celestes (sáculo) y rojas (glóbulos uni o múltiples). Estas estructuras se encuentran relacionadas con el almacenamiento de carbono en forma de lípidos y ácidos grasos, razón por la que se definen las vesículas como órganos de reserva del simbionte fúngico (Sieverding, 1983). Se observaron arbúsculos distribuidos a lo largo de la corteza, los que están involucrados en la transferencia bidireccional de nutrientes (Smith y Read, 1997).

Además de las estructuras mencionadas, se observaron otras hifas tabicadas, melanizadas y con numerosos microesclerocios del tipo de hongos endófitos septados oscuros (ESO) (Peterson et al., 2004; Figura 5).

Acorde con lo expuesto anteriormente, el presente estudio representa el primer antecedente de co-ocurrencia de ambos tipos de colonización en Topinambur (Helianthus tuberosus L.), tal como ocurre en la especie la familia Asteraceae, Smallanthus sonchifolius (Mercado et al., 2013), y en Miconia ioneura y Tibouchina paratropicade, especies de la fa- milia Melastomataceae (Urcelay et al., 2005). Todas estas especies procedentes del noroeste Argentino.

Se ha propuesto que estos hongos mejoran el funcionamiento de las plantas en ambientes áridos por las características morfo-anatómicas y algunos estudios experimentales, en virtud de su extensa red hifal, que aumenta el área de exploración del suelo, permitiendo a la mayoría de las plantas acceder a las fuentes de agua y nutrientes y mejorar así la supervivencia de las plantas hospedantes (Lugo et al., 2011), tal como sucede con las micorrizas MA (Glomus spp.) que contribuyen al crecimiento y la acumulación de cobre en girasol (Helianthus annuus) y favorecen los procesos de recuperación de suelos contaminados con desechos tóxicos (Castañón-Silva et al., 2013).

Las raíces presentaron un $97 \%$ de colonización micorrícica. La frecuencia de aparición de arbúsculos y de vesículas fue de $34 \%$ y $21 \%$ respectivamente. La frecuencia de aparición de microesclerocios de ESO alcanzó el 11\% de las raíces evaluadas.
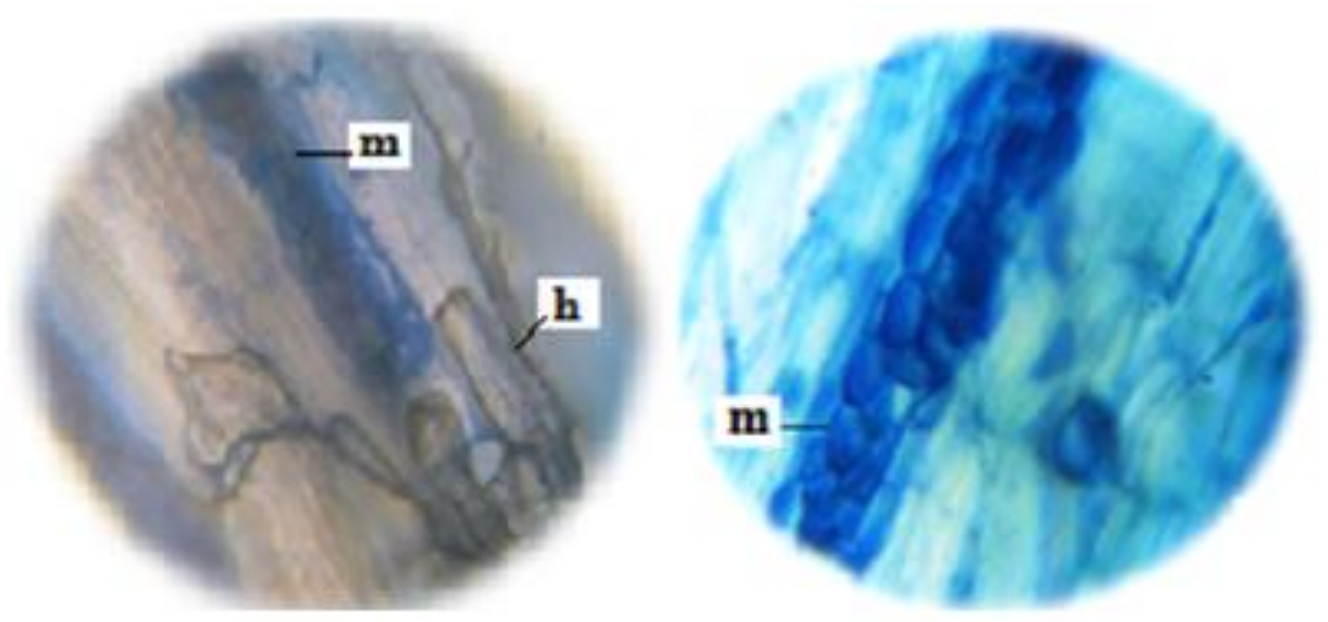

Figura 5. Hifas melanizadas (h) y microesclerocios (m) del tipo de hongos endófitos septados oscuros (ESO) en raicillas de topinambur (Helianthus tuberosus L.). Aumento de (40x). 
El alto nivel de colonización con hongos VA nos permite inferir que esta especie vegetal se comporta como micotrofa dependiente. Estos resultados sugieren que simbiontes fúngicos, micorrizas y endófitos septados oscuros nativos están presentes en el agroecosistema en estudio, y también parecen encontrarse en una concentración alta en el suelo, lo que estaría permitiendo la asociación con las raíces de topinambur favorecidas por el ambiente en el que se desarrollan y adaptadas a las condiciones del suelo.

Las raíces de Topinambur estuvieron simultáneamente colonizadas por ambos endófitos, hifas de micorrizas vesículo arbusculares y de septados oscuros, detectándose también la presencia de vesículas, arbúsculos típicos de micorrizas VA y de microesclerocios de ESO. El alto nivel de colonización fúngica con ocurrencia simultánea de micorrizas vesículo arbusculares y hongos ESO han sido observadas en todas las plantas estudiadas. En la Figura 6 se muestra la presencia de vesículas y de microesclerocios en una raicilla de topinambur, lo cual indica la colonización dual por micorrizas y endófitos septados oscuros. Esto también fue observado en diversos grupos de plantas, incluyendo helechos, mono y dicotiledóneas que se encuentran simultáneamente colonizados por micorrizas vesículo arbusculares (MVA) y endófitos septados oscuros (ESO) (Urcelay et al., 2005; Lugo et al., 2011; Lizárraga et al., 2015).

Se estima que estas interacciones microbianas con raíces de topinambur son microorganismos nativos debido a que el cultivo se realizó en un monte natural sin historial de cultivo previo, sumado a que es un cultivo no tradicional en la provincia de Catamarca y que es producido casi exclusivamente por un solo agricultor en la zona.

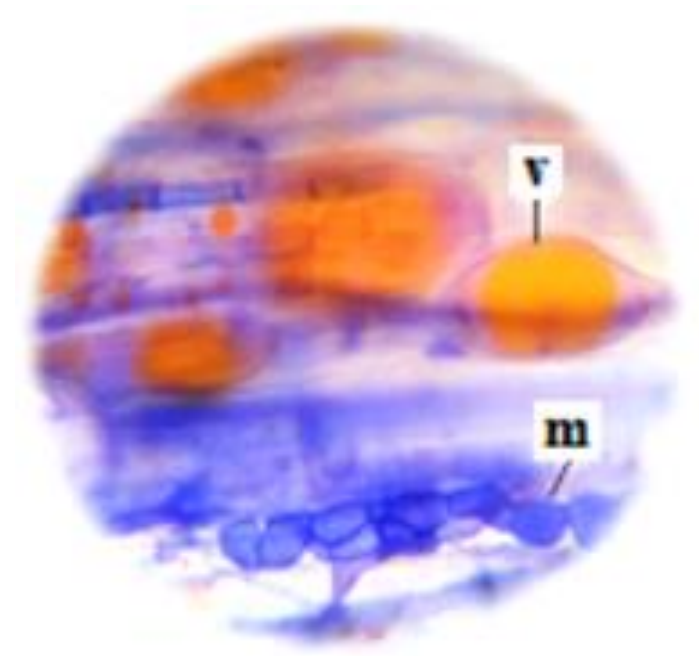

Figura 6. Vesículas (v) y microesclerocios (m) del tipo de hongos endófitos septados oscuros (ESO) en raicillas de topinambur (Helianthus tuberosus L.). Aumento de (40x).

\section{CONCLUSIONES}

Se describe por primera vez para Argentina, la asociación micorrícica en Topinambur $(\mathrm{He}$ lianthus tuberosus L.) con la co-ocurrencia de micorrizas vesículo arbusculares y endófitos septados oscuros nativos de Catamarca.

Se registra un alto nivel de colonización fúngica, con presencia de vesículas polimórficas, arbúsculos típicos de micorrizas VA y de hifas tabicadas, melanizadas con numerosos microesclerocios de ESO.

\section{REFERENCIAS BIBLIOGRÁFICAS}

1. Álvarez, C.; Scianca, C.; Barraco, M. 2008. Fertilización fosfatada e inoculación con micorrizas en girasol. Asagir. En: http://www. asagir.org.ar/descargar-archivo-158/archivos-e33c7ca860479c961c763742a9cd2e60; consulta: junio, 2017. 
2. Barrow, J. R.; Aaltonen, R. E. 2001. Evaluation of the internal colonization of Atriplex canescens (Pursh) Nutt. roots by dark septate fungi and the influence of host physiological activity. Mycorrhiza 11: 199 - 205. doi: 10.1007/s005720100111.

3. Barrow, J. R. 2003. Atypical morphology of dark septate fungal root endophytes of Bouteloua in arid southwestern U.S.A. rangelands. Mycorrhiza. 13(5):239 - 247. doi: 10.1007/s00572-003-0222-0.

4. Castañón-Silva, P.A.; Venegas-Urrutia, M. A.; Lobos-Valenzuela, M. G.; Gaete-Olivares, H. J. 2013. Influence of arbuscular mycorrhizal Glomus spp. On growth and accumulation of copper in sunflower $\mathrm{He}$ lianthus annuus L. Agrociencia. 47(4):309 - 317.

5. Cosgrove, D. R.; Oelke, D. A.; Doll, J. D.; Davis, D. W.; Undersander, D. J.; Oplinger, E. S. 1991. Topinambur. Alternative Field Crops Manual. Jerusalem artichoke. https://hort.purdue.edu/newcrop/afcm/ jerusart.html; consulta: junio, 2017.

6. Covacevich, F.; Echeverría, H. E.; Aguirrezabal, L. A. N. 2001. Comparación de dos técnicas de cuantificación de infección micorrícica. Ciencia del Suelo. 19(2):155 - 158.

7. Giovannetti, M.; Mosse, B. 1980. An evaluation of techniques for measuring vesicular- arbuscular infection in roots. New Phytologist. 84:489 - 500. doi: 10.1111/ j.1469-8137.1980.tb04556.x.

8. Harrison, M. J. 2005. Signaling in the arbuscular mycorrhizal symbiosis. Annu Rev Microbiol. 59:19 - 42. doi: 10.1146/ annurev.micro.58.030603.123749.

9. Jumpponen, A. 2001. Dark septate endophytes - are they mycorrhizal?. Mycorrhiza. 11:207 - 211. doi:10.1007/ s005720100112.
10. Jumpponen, A.; Trappe, J. M. 1998. Darkseptate root endophytes: a review with special reference to facultative biotrophic symbiosis. New Phytol. 140:295 - 310. doi: 10.1046/j.1469-8137.1998.00265.x.

11. Kirk, P. M.; Cannon, P. F.; David, J. C.; Stalpers, J. 2001. Ainsworth and Bisby's Dictionary of the Fungi. 9th ed. CAB International, Wallingford, UK. doi: https://doi. org/10.1016/S0024-2829(03)00055-0.

12. Lizarraga, S.V.; Ruiz, A.I.; Salazar, S.M.; Díaz Ricci, J.C.; Albornoz, P.L. 2015. Micorrizas vesículo-arbusculares, endófitos septados oscuros y anatomía radical en Fragaria ananassa var. Camino Real (Rosaceae), en la provincia de Tucumán, Argentina. Revista Agronómica del Noroeste Argentino. 35(1):11 - 17.

13. Lugo, M. A.; Giordano, P. G.; Urcelay, C.; Crespo, E. M. 2011. Colonización radical por endófitos fúngicos en Trithrinax campestris (Arecaceae) de ecosistemas semiáridos del centro de Argentina. Boletín de la Sociedad Argentina de Botánica. 46(3-4):213 - 222.

14. Martínez-Garcia, L.B. 2011. Micorrizas arbusculares en ecosistemas semiáridos. Respuesta a factores de estrés ambiental. Ecosistemas. 20(2-3):117 - 120.

15. Mercado, M. I.; Coll A., M. V.; Brandán De Weht, C. I.; Ponessa, G. I.; Grau, A. 2013. Arbuscular mycorrhizal associations and dark septate endophytes in Yacon (Smallanthus sonchifolius) and a wild relative (Smallanthus macroscyphus). Boletín de la Sociedad Argentina de Botánica. 48(2):193 - 200.

16. Newsham, K.K.; Fitter, A.H.; Watkinson, A.R. 1995. Multi-functionality and biodiversity in arbuscular rmycorrhizas. Trends in Ecology and Evolution. 10:407 $-411$. 
17. Parodi, G.; Pezzani, F. 2011. Micorrizas arbusculares en dos gramíneas nativas de Uruguay en áreas con y sin pastoreo. Agrociencia Uruguay. 15(2):1 - 10.

18. Pérez, G. 2012. Fertilizantes y microorganismos en el cultivo de girasol. INTA. Estación Experimental Agropecuaria Pergamino. En:http://inta.gob.ar/sites/default/files/script-tmp-fertilizantes_y_microorganismos_en_el_cultivo_de_gira.pdf; consulta: junio, 2017.

19. Pérez, C. A.; Vertel, M. M. 2010. Evaluación de la colonización de micorrizas arbusculares en pasto Bothriochloa pertusa (L) A. Camus. Revista MVZ Córdoba. 15(3):2165 $-2174$.

20. Peterson, R. L.; Massicote, H. B.; Melville, L. H. 2004. Dark septate fungal endophytes. In P.B. CAVERS (ed.), Mycorrhizas: Anatomy and cell biology. NRC. Ottawa. 145 - 153p.

21. Phillips J. M.; Hayman, D. S. 1970. Improved procedures for clearing roots and staining parasitic and vesicular-arbuscular mycorrhizal fungi for rapid assessment of infection. Trans. Br. Mycol. Soc. 55:158 - 161. doi: 10.1016/S0007-1536(70)80110-3.

22. Rebora, C. 2008. Topinambur (Helianthus tuberosus L.): usos, cultivo y potencialidad en la región de Cuyo. Horticultura Argentina. (63):27 - 37.

23. Sarasola, A.; Rocca, M. 1975. Fitopatología. Curso moderno. Tomo IV. Editorial Hemisferio Sur. Buenos Aires. 191 - 192p.

24. Schübler, A.; Schwarzott, D.; Walker C. 2001. A new fungal phylum the Glomeromycota: phylogeny and evolution. Mycological Research. 105:1413 - 1421. doi: $10.1017 /$ S0953756201005196.

25. Selosse, M. A.; Richard, F.; He, X.; Simard, S. W. 2006. Mycorrhizal networks: des liaisons dangereuses?. Trends Ecol Evil. 21:621 - 628. doi: 10.1016/j. tree.2006.07.003.
26. Sieverding, E. 1983. Proyecto Micorriza. Centro Internacional de Agricultura Tropical. Cali. Colombia. 121p.

27. Smith, S. E.; Read, D. J. 1997. Chapter 2. The development of infection and anatomy of vesicular-arbuscular mycorrhizas. In "Mycorrhizal symbiosis". 2nd ed. Academic Press, London. 32 - 63p.

28. Urcelay, C.; Tecco, P. A.; Chiarini, F. 2005. Micorrizas arbusculares del tipo 'Arum' y 'Paris' y endófitos radicales septados oscuros en Miconia ioneura y Tibouchina paratropica (Melastomataceae). Boletín de la Sociedad Argentina de Botánica. 40(3-4):151 - 155.

29. Wang, B.; Qiu, Y. L. 2006. Phylogenetic distribution and evolution of mycorrhizas in land plants. Mycorrhizahello. 16(5):299 363.

30. Verna, L.C.; Herrero, F.J. 1952. Micología. Morfología, biología, experimentación. El Ateneo. Buenos Aires. 209 - 210p. 